\title{
DIFFERENCES IN MRSA PREVALENCE AND RESISTANCE PATTERNS IN A TERTIARY CENTER BEFORE AND AFTER JOINING AN INTERNATIONAL PROGRAM FOR SURVEILLANCE OF ANTIMICROBIAL RESISTANCE
}

\author{
OLIVERA DJURIC ${ }^{1,4} *$, SNEZANA JOVANOVIC ${ }^{2}$, BRANKA STOSOVIC ${ }^{2}$, \\ TANJA TOSIC ${ }^{2}$, Milica JOVANOVIC ${ }^{2}$, NAOMI NARTEY ${ }^{4}$, JOVANA TODOROVIC ${ }^{3,4}$ \\ and LJILJANA MARKOVIC-DENIC ${ }^{1,4}$ \\ ${ }^{1}$ Institute of Epidemiology, Faculty of Medicine, University of Belgrade, Belgrade, Serbia \\ ${ }^{2}$ Department of Microbiology, Clinical Centre of Serbia, Belgrade, Serbia \\ ${ }^{3}$ Institute of Social Medicine, Faculty of Medicine, University of Belgrade, \\ Belgrade, Serbia \\ ${ }^{4}$ Faculty of Medicine, University of Belgrade, Belgrade, Serbia
}

(Received: 23 May 2016; accepted: 25 August 2016)

\begin{abstract}
Methicillin-resistant Staphylococcus aureus (MRSA) emerged as one of the most important causes of hospital-acquired bloodstream infections (BSIs), especially the multidrug resistant clones. The aim of the present study was to compare prevalence and resistance patterns of MRSA bacteremia in the major tertiary-care academic and referral center in Serbia before and after implementing an active antimicrobial resistance (AMR) surveillance. Laboratory-based before-after study was conducted during a two-year period (January 2012 to December 2013) in Clinical Centre of Serbia. Isolation and identification of bacterial strains were done following standard microbiological procedures. During the AMR surveillance, nearly twice more bloodstream samples were collected compared to the year without surveillance $(1,528 \mathrm{vs}$. 855). In total, 43 isolates of MRSA were identified. MRSA was significantly more prevalent during the AMR surveillance compared to the previous year [14 (66.7\%) to $29(76.3 \%) ; P=0.046]$. During the AMR surveillance, MRSA more frequently originated from medical departments compared to intensive care unit, surgical department, and internal medicine $(P=0.027)$ indicating increasing MRSA infections in patients with less severe clinical condition and no apparent risk factors. Higher prevalence of MRSA and its lower susceptibility to erythromycin were revealed by implementation of active AMR surveillance, which may reflect more thoughtful collection of bloodstream samples from patients with suspected BSI.
\end{abstract}

*Corresponding author; E-mail: oliveradjuric87@gmail.com 
Keywords: HA-MRSA, bacteremia, surveillance, antimicrobial resistance

\section{Introduction}

Methicillin-resistant Staphylococcus aureus (MRSA) is one of the most important pathogens causing various infections in hospitals and community. Since its first detection in 1961, MRSA still imposes a priority in prevention and management of hospital-acquired infections (HAIs), due to health and financial burden it delivers, especially in countries with limited resources. According to the last ECDC technical report, MRSA accounted for the $44 \%$ of all HAIs and $12 \%$ of all bacteremia in the EU states, Iceland, and Norway, while causing 22\% attributable extra deaths and $41 \%$ of in-hospital extra days [1]. Considering the financial burden, median attributable hospital charge for MRSA bacteremia in the USA amounts to US\$ 6,916 [2], while in Europe MRSA bloodstream infections (BSIs) account for EUR 380 million extra costs annually [1].

Although moderate declining of hospital-acquired MRSA (HA-MRSA) bacteremia was recently observed in a few European countries, the United States and Australia [3-5], this pathogen is the most common single multidrug resistant (MDR) cause of HAIs in Europe, the United States, Asia, and Brazil [6-9]. The European/EEA population-mean of invasive HA-MRSA infections in 2013 was $18.0 \%$ [10]; however, these percentages vary greatly within a continent, from 0 in Iceland up to $64.5 \%$ in Romania, indicating major differences in epidemiology of MRSA bacteremia, while data from the non-EU states of the Balkan region remain underrepresented.

Active surveillance is the first and the vital step in the battle against MRSA since it depicts the real extent of pathogen distribution and its antimicrobial resistance (AMR) in a particular medical setting. The importance of wellorganized programs for infection surveillance and control was stressed out even in 60s and 70s by the Center for Disease Control (CDC) [11]. By now most of the countries have implemented some kind of infection control program and current decrease in MRSA bacteremia in most of them was mainly attributed to the interventions or action plans to prevent these infections [12, 13]. However, it is hard to distinguish what is the contribution of the active surveillance per se and its impact on the clinical susceptibility data.

Serbia established national committee for infection control in 2003, however, has not implemented neither general/MRSA-specific infection control programs nor the national AMR action plan so far due to consistently high MRSA rates. According to the last national point prevalence study of HAIs conducted in $2010,51 \%$ of all $S$. aureus infections were caused by MRSA strains [14]. In 2013, Serbia has joined the CAESAR (Central Asian and Eastern 
European Surveillance of Antimicrobial Resistance) network, which is the network of national AMR surveillance systems in all countries of the region that are not part of the EARS-Net (European AMR Surveillance Network) coordinated by ECDC, with the purpose to report AMR data on eight invasive bacteria of public health and clinical importance, among which, S. aureus [15].

Pursuant to this, the aim of the present study was to compare prevalence and resistance patterns of MRSA bacteremia before and after the implementation of active microbiological surveillance in the setting with no national actions plans and interventions regarding MRSA-related infection control.

\section{Materials and Methods}

National AMR surveillance network

The national AMR surveillance in Serbia, as a part of CAESAR network, has started in January 2013. Detailed methods of data collection and quality control are presented in CAESAR annual report of AMR [15]. In brief, it comprises 14 patient-care laboratories that serve secondary and tertiary care hospitals and covers nine out of 24 regions in Serbia, which represents more than $40 \%$ of the entire population. The representative AMR results, based on routine samples of blood and cerebrospinal fluid, were collected by labs and forwarded to the Reference Laboratory. Collected data comprised patients' background information (an isolate record form) and antimicrobial susceptibility test results of invasive isolates of $S$. aureus, Enterococcus faecalis, Enterococcus faecium, Streptococcus pneumoniae, Escherichia coli, Klebsiella pneumoniae, Pseudomonas aeruginosa, and Acinetobacter spp. Data were submitted to the reference laboratory either as a paper record form or electronically (by an e-mail). Reference laboratory used WHONET 5.6 for data collection and analysis.

\section{Study design and setting}

A laboratory-based before-after study was conducted in a tertiary-care center (Clinical Centre of Serbia - CCS) in Belgrade, the capital of Serbia, during a twoyear period (January 2012 to December 2013) in order to compare occurrence of bacteremia caused by MRSA and other bacteria of public health importance before and after the initiation of national AMR surveillance, as well as to compare susceptibility patterns of MRSA strains with respect to AMR surveillance implementation. Prior to beginning of the AMR surveillance, the clinicians were 
trained and encouraged to take blood samples from all patients presenting with signs or symptoms of BSI, i.e., fever $\left(>38{ }^{\circ} \mathrm{C}\right)$, chills or hypotension, as recommended by Centers for Disease Control and Prevention/National Healthcare Safety Network (CDC/NHSN) [16].

The CCS is the largest tertiary healthcare and academic institution in Serbia comprising 28 departments and 3,500 beds. It serves the entire population of Serbia, and performs nearly 6 million laboratory services annually.

\section{Data collection}

Data for the surveillance year (January 1 to December 31, 2013) were collected from the standardized isolate record forms, which comprised microbiological data and patient's demographic information and were prospectively collected by the affiliated patient-care microbiological laboratories of CCS (Emergency Centre, Clinic for Infectious and Tropic Diseases and Polyclinic) and forwarded to the principal laboratory (Polyclinic). Data on patients' characteristics were age, sex, and the department at the onset of bacteremia (surgery department - SD; intensive care unit - ICU, internal medicine or other medical departments). For the purpose of this study, we extracted surveillance data of antimicrobial susceptibility test results of $S$. aureus isolates obtained from blood cultures of adult patients hospitalized in various departments of the CCS. Only primary blood culture per patient was analyzed and the first invasive isolate per culture was reported. Collected data was gathered and checked for consistency with protocol agreed.

For the year previous to surveillance (January 1 to December 31, 2012), susceptibility data of MRSA strains was collected retrospectively from the laboratory protocols, which comprised microbiological data and patients' demographic data. For the purpose of better comparability, only the same laboratories which used to provide samples to the principal laboratory in both years and cover the same clinical wards were included in the analysis. Susceptibility data from 2012 that was not obtained using the same laboratory standards and/or not in compliance with EARS-Net recommendations were excluded from the analysis.

\section{Microbiological assessment}

In all laboratories, isolation and identification of bacterial strains were done following standard microbiological procedures. Antimicrobial susceptibility was estimated by using Kirby-Bauer disc diffusion and using Vitek2 Automated 
System (bioMérieux, Marcy-I'Etoile, France). The spectrum of antimicrobial drugs reported is in accordance with EARS-Net. Zone diameter was measured and interpreted according to the Clinical and Laboratory Standards Institute (CLSI) guidelines [17]. Strains which showed intermediate susceptibility (I) and resistance $(\mathrm{R})$ to the specific antibiotic were considered resistant $(\mathrm{R})$. Methicillin resistance of $S$. aureus was determined by using Kirby-Bauer disc diffusion method with $30 \mu \mathrm{g}$ cefoxitin disc (Becton Dickinson, Sparks, USA). A zone size of $\geq 22 \mathrm{~mm}$ was considered sensitive (S) and $\leq 21 \mathrm{~mm}$ was considered resistant (R). Minimum inhibitory concentrations were determined by E-test and results were interpreted according to CLSI clinical breakpoints.

\section{Statistical analysis}

Descriptive statistics was used to express the frequency of bacterial isolates and their susceptibility patterns. Numbers and percentages were used for categorical and ordinal data. Chi-square test or Fisher exact test was applied for two-group comparison of categorical data. All statistical tests were two-sided and were performed at the 5\% significance level. Statistical analysis was performed by using the IBM SPSS Statistics version 20.0 software (IBM Corp., Armonk, NY, USA).

\section{Results}

Flowchart of the process of blood cultures gathering during the two consequent years, before and during the first year of national AMR surveillance, is presented numerically in Figure 1. During the active surveillance, approximately one and a half times more samples were collected, starting from the blood culture bottles obtained from patients with suspected BSI and consequently to the primary isolates with positive microbial growth. In both years, Gram-positive bacteria were predominantly cultured (57\% and 68\%), followed by Gram-negative bacteria ( $23 \%$ and $21 \%$ ) and fungi $(20 \%$ and $11 \%)$. However, the all three types of causative agents were significantly more prevalent during the AMR surveillance compared to the previous year $(P<0.001)$ (Figure 2).

The distributions of the invasive bacteria of public health importance that are subjected to annual reporting to CAESAR, as well as the percent of changes during the two years of follow up (before and during the AMR surveillance), are shown in Table I. Acinetobacter spp. and K. pneumoniae were the most prevalent bacteria in both years, followed by E. faecium and $S$. aureus. P. aeruginosa, $S$. pneumoniae, and E. coli were less frequently isolated than the above-mentioned 


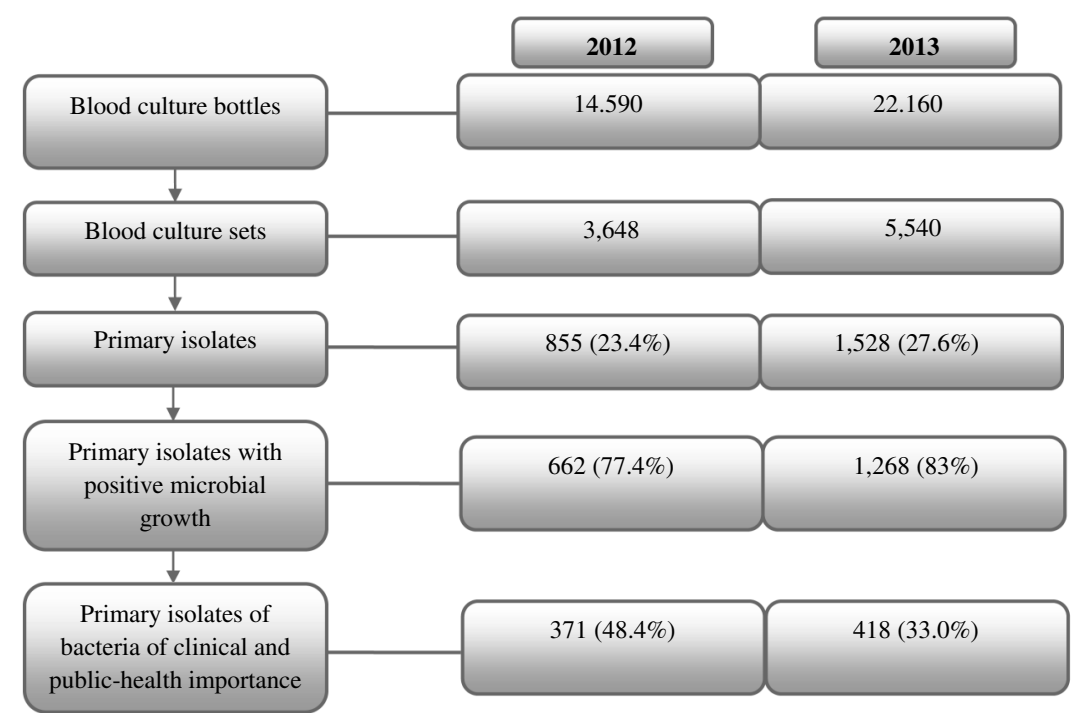

Figure 1. Flowchart of the blood cultures collection before (2012) and during (2013) the AMR surveillance

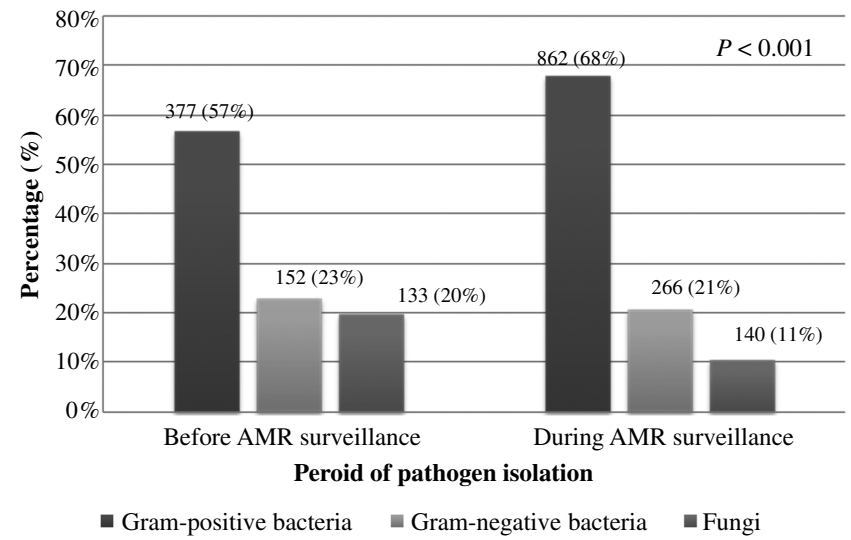

Figure 2. Types of causative agents of bloodstream infections before (2012) and during (2013) the AMR surveillance

bacteria, before and during the AMR surveillance. When comparing those two years, significant change in frequency was observed for four out of eight bacteria. While frequency of Acinetobacter spp. and E. faecalis decreased from $51 \%$ to $41.9 \%$ and from $6.1 \%$ to $3.3 \%$, respectively, K. pneumoniae and S. aureus 
Table I. Distribution of bacteria of public-health importance before and during the AMR surveillance

\begin{tabular}{|c|c|c|c|c|}
\hline & $\begin{array}{l}\text { Before AMR } \\
\text { surveillance }\end{array}$ & $\begin{array}{l}\text { During AMR } \\
\text { surveillance }\end{array}$ & $\begin{array}{l}\text { Change } \\
(\%)\end{array}$ & $P$-value \\
\hline Acinetobacter spp. & $192(51.8)$ & $175(41.9)$ & -9.9 & $<0.001$ \\
\hline Klebsiella pneumoniae & $63(16.9)$ & $111(26.6)$ & 9.7 & 0.001 \\
\hline Enterococcus faecium & 43 (11.6) & $40(9.6)$ & -2.0 & 0.363 \\
\hline Enterococcus faecalis & $23(6.1)$ & $14(3.3)$ & -2.8 & 0.046 \\
\hline Staphylococcus aureus & $21(5.7)$ & $38(9.1)$ & 3.4 & 0.026 \\
\hline $\begin{array}{l}\text { Methicillin-resistant } \\
\text { S. aureus }\end{array}$ & $14(66.7)$ & $29(76.3)$ & 9.6 & 0.046 \\
\hline $\begin{array}{l}\text { Methicillin-sensitive } \\
\text { S. aureus }\end{array}$ & $7(33.3)$ & $9(23.7)$ & -9.6 & 1.000 \\
\hline Pseudomonas aeruginosa & $11(2.9)$ & $19(4.5)$ & 1.6 & 0.529 \\
\hline Streptococcus pneumoniae & $10(2.7)$ & $1(0.2)$ & 2.5 & 0.062 \\
\hline Escherichia coli & $8(2.2)$ & $20(4.8)$ & 2.6 & 0.352 \\
\hline Total & $371(100.0)$ & $418(100.0)$ & & \\
\hline
\end{tabular}

Note: Bold values are statistically significant $(P<0.05)$.

significantly increased during the AMR surveillance. Interestingly, increment of $S$. aureus has occurred due to the increase of methicillin-resistant strains [14 (66.7\%) to $29(76.3 \%) ; P=0.046]$.

In a year prior to AMR surveillance, MRSA strains were predominantly isolated from males and middle-aged and elderly inpatients, while during the surveillance year MRSA strains were more frequently isolated from females and middle-age patients (Table II). However, medical wards from which MRSA

Table II. Characteristics and hospitalization wards of inpatients with MRSA bacteriaemia at the moment of blood culture collection

\begin{tabular}{|c|c|c|c|}
\hline & $\begin{array}{l}\text { Before AMR surveillance } \\
\qquad(n=14)\end{array}$ & $\begin{array}{l}\text { During AMR surveillance } \\
\qquad(n=29)\end{array}$ & $P$-value \\
\hline \multicolumn{4}{|l|}{ Gender, $n(\%)$} \\
\hline Male & $8(57.1)$ & $12(41.4)$ & 0.331 \\
\hline Female & $6(42.9)$ & $17(58.6)$ & \\
\hline \multicolumn{4}{|l|}{ Age, $n(\%)$} \\
\hline $20-65$ & $6(42.9)$ & $16(55.2)$ & 0.640 \\
\hline$>65$ & $6(42.9)$ & $11(37.9)$ & \\
\hline Missing & $2(14.3)$ & $2(6.9)$ & \\
\hline \multicolumn{4}{|l|}{ Hospital ward, $n(\%)$} \\
\hline ICU & $7(50.0)$ & $8(27.6)$ & 0.027 \\
\hline Surgery & $2(14.3)$ & $7(24.1)$ & \\
\hline Internal department & $3(21.4)$ & $3(10.3)$ & \\
\hline Medical departments & $2(14.3)$ & $11(37.9)$ & \\
\hline
\end{tabular}

Note: MRSA, methicillin-resistant Staphylococcus aureus; ICU, intensive care unit. Bold value is statistically significant $(P<0.05)$. 
isolates originated significantly changed in favor of medical departments (37\%), while isolation of MRSA in the ICU and internal department reduced by double $(P=0.027)$.

Results of antimicrobial susceptibility testing and change in fraction of resistant strains of MRSA isolates before and during the AMR surveillance are shown in Table III. In both years, MRSA was highly resistant to gentamicin and ciprofloxacin. Moreover, before the AMR surveillance all MRSA isolates were resistant to rifampicin as well, and moderately resistant to clindamycin and erythromycin, while completely susceptible to sulfamethoxazole-trimethoprim (SMX-TMP). This pattern had changed in terms that during the AMR surveillance implementation resistance to rifampicin significantly decreased $(100 \%-48.3 \%$; $P=0.001)$ while resistance to erythromycin increased $(25 \%-62.1 \% ; P=0.043)$. Importantly, in the year prior to AMR surveillance, only one MRSA strain resistant to vancomycin and linezolid was isolated in the entire CCS, while in AMR surveillance year all MRSA isolates were susceptible to those two last-line antibiotics.

Table III. Antibiotic susceptibility of MRSA strains to other antibiotics before and during the AMR surveillance

\begin{tabular}{|c|c|c|c|c|c|c|c|c|}
\hline \multirow{2}{*}{$\begin{array}{l}\text { Antimicrobial } \\
\text { class/agent }\end{array}$} & \multicolumn{3}{|c|}{$\begin{array}{l}\text { Before AMR surveillance } \\
\qquad(n=14)\end{array}$} & \multicolumn{3}{|c|}{$\begin{array}{l}\text { During AMR surveillance } \\
\qquad(n=29)\end{array}$} & \multirow{2}{*}{$\begin{array}{c}\text { Change } \\
(\%)\end{array}$} & \multirow[b]{2}{*}{$P$-value } \\
\hline & $n$ & $n(\%) \mathrm{S}$ & $n(\%) \mathrm{R}$ & $n$ & $n(\%) \mathrm{S}$ & $n(\%) \mathrm{R}$ & & \\
\hline \multicolumn{9}{|c|}{ Aminoglycosides } \\
\hline $\begin{array}{l}\text { Gentamicin } \\
\text { Glycopeptides }\end{array}$ & 14 & $2(14.3)$ & $12(85.7)$ & 18 & $0(0.0)$ & $18(100.0)$ & 14.3 & 0.183 \\
\hline \multicolumn{8}{|c|}{ Fluoroquinolones } & 0.293 \\
\hline $\begin{array}{l}\text { Ciprofloxacin } \\
\text { Ansamycins }\end{array}$ & 14 & $1(7.1)$ & $13(92.9)$ & 25 & $3(12.0)$ & $22(88.0)$ & -4.9 & 1.000 \\
\hline $\begin{array}{l}\text { Rifampin/ } \\
\text { Rifampicin }\end{array}$ & 14 & $0(0.0)$ & $14(100.0)$ & 29 & $15(51.7)$ & $14(48.3)$ & -51.7 & 0.001 \\
\hline \multicolumn{9}{|l|}{ Lincosamides } \\
\hline $\begin{array}{l}\text { Clindamycin } \\
\text { Macrolides }\end{array}$ & 14 & $9(64.3)$ & $5(35.7)$ & 28 & $10(35.7)$ & $18(64.3)$ & 28.6 & 0.107 \\
\hline $\begin{array}{l}\text { Erythromycin } \\
\text { Oxazolidinones }\end{array}$ & 12 & $9(75.0)$ & $3(25.0)$ & 29 & $11(37.9)$ & $18(62.1)$ & 37.1 & 0.043 \\
\hline Linezolid & 14 & $13(92.2)$ & $1(7.1)$ & 27 & $27(100.0)$ & $0(0.0)$ & -7.1 & 0.341 \\
\hline Folate pathway & nhibi & & & & & & & \\
\hline SMX-TMP & 14 & $14(100.0)$ & $0(0.0)$ & 25 & $21(84.0)$ & $4(16.0)$ & 16.0 & 0.277 \\
\hline
\end{tabular}

Note: MRSA, methicillin-resistant Staphylococcus aureus; SMX-TMP, sulfamethoxazole-trimethoprim; $\mathrm{S}$, susceptible; $\mathrm{R}$, resistant. Bold values are statistically significant $(P<0.05)$.

${ }^{\mathrm{a}}$ Fisher exact test. 


\section{Discussion}

Over the course of antibiotic era, MRSA emerged as one of the most important causes of nosocomial infections, especially the MDR clones, mainly due to adverse outcomes and limited therapeutic options. Glycopeptides and recently developed antibiotic linezolid are currently the last line antimicrobials, which remain effective against HA-MRSA. Constant and accurate monitoring of its prevalence and resistance patterns is, therefore, a necessity.

The main finding of the present surveillance study is substantially higher prevalence of $S$. aureus isolated during the active AMR surveillance period compared to previous year without surveillance, which more importantly, was attributable to the increase of methicillin-resistant strains. This $10 \%$ increase (from $66.7 \%$ to $76.3 \%$ ) is worrisome given the already high rates of MRSA infections in CCS, which are therewithal, higher than the overall MRSA rates in Serbia in 2013 $(42 \%)$ as well as in other four countries of the Region, which have submitted their AMR data to the CAESAR (The former Yugoslav Republic of Macedonia, 41\%; Belarus, 35\%; Turkey, 26\%; and Switzerland, 5\%) [15]. Of note is that of the listed countries only Turkey and The former Yugoslav Republic of Macedonia have the National AMR action plan developed.

Although surveillance data more objectively represents current situation regarding MRSA infection in CCS, its generalizability to the entire country and comparability to other countries are doubtful since the CCS is a tertiary care hospital indebted for the most severe cases and that, consequently, majority of MRSA originated from ICU and SD. Even though the pre-surveillance department-distribution of MRSA in our study (50\% ICU and 14\% SD) is similar to the results reported in Romanian teaching hospital and Macedonian Clinical Centre $[18,19]$, these rates almost equalized during the surveillance year (27.6\% ICU and $24.1 \%$ SD) while the medical departments featured predominantly $(37.9 \%)$. It is hard to find the solid grounds that underpin such a difference within a single center as well as to answer whether the surveillance play an important role. Presumably, more heedful requesting of blood samples from inpatients with less severe clinical condition in medical departments contributed to the increased MRSA rates obtained from these wards. This is also in a line with recent reports on MRSA infections emerging among patients with no evident risk factors [20]. Frequent transfer of inpatients between ICU and SD may have contributed to the similar MRSA rates in these wards. It is also shown that ICU and SD are the wards, which most commonly use antibiotics for prophylactic purposes in Vojvodina region in Serbia [21].

Regarding the AMR, we found high resistance to gentamicin, ciprofloxacin, and clindamycin in both years, prior and during the active surveillance. These rates 
are more than twice higher than reported to CAESAR for the entire country but similar to single clinical centers in neighboring countries, Romania and Macedonia [18, 19]. Only rifampicin and erythromycin significantly changed in our study, first lowered and second increased during the AMR surveillance. Irrational use of antibiotics is one of the reasons for such a high prevalence of MRSA and its AMR rates since Serbia is one of the southern and eastern European countries with the highest rates of total antimicrobial consumption while second in utilization of 1st generation cephalosporins, macrolides, and tetracycline [22].

Clonal and patients healthcare workers (HCWs) dissemination of MRSA is another likely reason for the non-lowering MRSA rates after surveillance initiation. Recent cross-sectional studies of MRSA carriage in CCS showed high MRSA nasal and throat carriage rates, $11.8 \%$ in patients, and $7.6 \%$ in $\mathrm{HCWs}$, and predominance of CC5-MRSA-SCCmecI MRSA lineage in emergency and medical departments $[23,24]$. Moreover, it was recently shown that genetic lineages characteristic for community-acquired MRSA (CA-MRSA) (SCCmec types IV and V) are more frequent than HA-MRSA lineages (SCCmec types I, II, and III) in both hospitalized and healthy patients in Pomoravlje region in Serbia, indicating transfer and adaptation of CA-MRSA strains to hospital environment and acquiring additional resistance due to inadequate hygiene measures and selective pressure of antibiotics [25]. Molecular typing surveillances are usually singlecenter-based due to considerable financial and human resources they require. However, as demonstrated by few countries, including molecular typing data in on-going AMR surveillance can delineate new molecular sequence types and allow better understanding of molecular epidemiology of MRSA [26, 27].

While nationally and internationally conducted surveys depict global spread and burden of the infection, locally derived ones are crucial in updating clinicians and guiding them toward the most adequate empirical therapy [28, 29]. One of the advantages of well-organized surveillance is larger number of collected samples, which doubtlessly highlight which pathogen, when isolated, is the biggest threat [30]. Significant increase in collected blood-culture bottles and detection of MRSA after initiation of national surveillance in our study are direct effects of more systematic data collection and reporting in laboratories during the active surveillance. However, laboratories were not provided with the data regarding the origin of the BSIs, which is important for understanding the epidemiology of MRSA bacteremia.

To the best of our knowledge, this is the first study to delineate the prevalence and resistance patterns of bloodstream MRSA isolates in the entire CCS with respect to initiation of the active AMR surveillance. This kind of pathogen monitoring is crucial especially in countries like Serbia, where due to limited resources all efforts are curative-oriented and little or no investment is 
allocated to preventative measures [31]. In such a setting, it has multiple contributions by substantially influencing the image of AMR extent which can increase awareness among clinicians and public health authorities and more importantly it can allow wiser and more justified allocation of resources to prevent HAIs.

The main limitation of the study refers to the uncontrolled before-after design and involves the time trend bias which can lead to overestimation of the influence of the implemented survey. It implies uncertainty whether changes occurred due to surveillance itself or are the consequence of secular trends or sudden changes [32]. Prevalence and department distribution of MRSA isolates are likely influenced by more systematic data collection during the AMR surveillance, on the other hand, AMR rates and patterns are the effect of more complex interactions between microbial genetics and antibiotic selective pressure, which is required to be assessed as well. However, results of an active, standardized survey of MRSA AMR are more representative of current situation regarding MRSA frequency and resistance than unsystematic data collection.

\section{Acknowledgement}

This study was financially supported by the Ministry of Education, Science and Technological Development, Republic of Serbia (Project No. 175046).

\section{Conflict of Interest}

The authors declare no conflict of interest related to this work.

\section{References}

1. Centers for Disease Prevention and Control: The bacterial challenge: Time to react. ECDC/EMEA Joint Technical Report. European Centre for Disease Prevention and Control, Stockholm, Sweden (2009). Available at http://ecdc.europa.eu/en/publications/ Publications/0909_TER_The_Bacterial_Challenge_Time_to_React.pdf

2. Ippolito, G., Leone, S., Lauria, F. N., Nicastri, E., Wenzel, R. P.: Methicillin-resistant Staphylococcus aureus: The superbug. Int J Infect Dis 14, S7-S11 (2010).

3. de Kraker, M. E., Jarlier, V., Monen, J. C., Heuer, O. E., van de Sande, N., Grundmann, H.: The changing epidemiology of bacteraemias in Europe: Trends from the European Antimicrobial Resistance Surveillance System. Clin Microbiol Infect 19, 860-868 (2013). 
4. Centers for Disease Prevention and Control: Active bacterial core surveillance report, Emerging Infections Program Network, methicillin-resistant Staphylococcus aureus, 2012 (2012). Available at https://www.cdc.gov/abcs/reports-findings/survreports/ mrsa14.pdf

5. Mitchell, B. G., Collignon, P. J., McCann, R., Wilkinson, I. J., Wells, A.: A major reduction in hospital-onset Staphylococcus aureus bacteremia in Australia - 12 years of progress: An observational study. Clin Infect Dis 59, 969-975 (2014).

6. Kock, R., Becker, K., Cookson, B., van Gemert-Pijnen, J. E., Harbarth, S., Kluytmans, J., Mielke, M., Peters, G., Skov, R. L., Struelens, M. J., Tacconelli, E., Navarro Torne, A., Witte, W., Friedrich, A. W.: Methicillin-resistant Staphylococcus aureus (MRSA): Burden of disease and control challenges in Europe. Euro Surveill 15, 19688 (2010).

7. Grundmann, H., Aires-de-Sousa, M., Boyce, J., Tiemersma, E.: Emergence and resurgence of methicillin-resistant Staphylococcus aureus as a public-health threat. Lancet $\mathbf{3 6 8}$, 874-885 (2006).

8. Zhao, C., Sun, H., Wang, H., Liu, Y., Hu, B., Yu, Y., Sun, Z., Chu, Y., Cao, B., Liao, K., Lei, J., Hu, Z., Zhang, L., Zhang, X., Xu, Y., Wang, Z., Chen, M.: Antimicrobial resistance trends among 5608 clinical Gram-positive isolates in China: Results from the GramPositive Cocci Resistance Surveillance program (2005-2010). Diagn Microbiol Infect Dis 73, 174-181 (2012).

9. Carvalho, K. S., Mamizuka, E. M., Gontijo Filho, P. P.: Methicillin/Oxacillin-resistant Staphylococcus aureus as a hospital and public health threat in Brazil. Braz J Infect Dis 14, 71-76 (2010).

10. European Centre for Disease Prevention and Control: Antimicrobial resistance surveillance in Europe 2013. Annual Report of the European Antimicrobial Resistance Surveillance Network (EARS-Net) (2014). Available at http://ecdc.europa.eu/en/publications/ Publications/antimicrobial-resistance-surveillance-europe-2013.pdf

11. World Health Organization: WHO global strategy for containment of antimicrobial resistance (2001).

12. Rampling, A., Wiseman, S., Davis, L., Hyett, A. P., Walbridge, A. N., Payne, G. C., Cornaby, A. J.: Evidence that hospital hygiene is important in the control of methicillinresistant Staphylococcus aureus. J Hosp Infect 49, 109-116 (2001).

13. Chalfine, A., Kitzis, M. D., Bezie, Y., Benali, A., Perniceni, L., Nguyen, J. C., Dumay, M. F., Gonot, J., Rejasse, G., Goldstein, F., Carlet, J., Misset, B.: Ten-year decrease of acquired methicillin-resistant Staphylococcus aureus (MRSA) bacteremia at a single institution: The result of a multifaceted program combining cross-transmission prevention and antimicrobial stewardship. Antimicrob Resist Infect Control 1, 18 (2012).

14. Markovic-Denic, L. S. V., Mijovic, B., Cosic, G., Tiodorovic, B., Carevic, B.: Third national study of hospital-acquired infections. Ministry of Health of Republic of Serbia (2010).

15. Central Asian and Eastern European Surveillance of Antimicrobial Resistance (CAESAR) Network: Annual report on antimicrobial resistance, 2014 (2014).

16. Horan, T. C., Andrus, M., Dudeck, M. A.: CDC/NHSN surveillance definition of health care-associated infection and criteria for specific types of infections in the acute care setting. Am J Infect Control 36, 309-332 (2008).

17. Clinical and Laboratory Standards Institute: Performance standards for antimicrobial susceptibility testing: 23rd informational supplement. CLSI document, M100-S23 (2013). 
18. Szekely, E., Lorinczi, L., Bilca, D., Fodor, E., Soki, J., Sabau, M.: Incidence, antibiotic resistance and clonal relations of MRSA strains isolated from a Romanian university hospital. Acta Microbiol Immunol Hung 55, 1-13 (2008).

19. Cekovska, Z., Panovski, N., Petrovska, M., Kristof, K., Rozgonyi, F.: Incidence of Staphylococcus aureus isolated from patients treated at the Clinical Center of Skopje, Macedonia, with special attention to MRSA. Acta Microbiol Immunol Hung 52, 373-384 (2005).

20. Deresinski, S.: Methicillin-resistant Staphylococcus aureus: An evolutionary, epidemiologic, and therapeutic odyssey. Clin Infect Dis 40, 562-573 (2005).

21. Cosic, G. D. J., Petrovic, M., Krtinic, G., Karac, T., Jandric-Kocic, J., Markovic-Denic, L.: The most frequent hospital-acquired infections related to medical interventions in hospitals in Vojvodina region. Arch Biol Sci 66, 523-525 (2014).

22. Versporten, A., Bolokhovets, G., Ghazaryan, L., Abilova, V., Pyshnik, G., Spasojevic, T., Korinteli, I., Raka, L., Kambaralieva, B., Cizmovic, L., Carp, A., Radonjic, V., Maqsudova, N., Celik, H. D., Payerl-Pal, M., Pedersen, H. B., Sautenkova, N., Goossens, H., on behalf of WHO/Europe-ESAC Project Group: Antibiotic use in eastern Europe: A cross-national database study in coordination with the WHO Regional Office for Europe. Lancet Infect Dis 14, 381-387 (2014).

23. Cirkovic, I., Stepanovic, S., Skov, R., Trajkovic, J., Grgurevic, A., Larsen, A. R.: Carriage and genetic diversity of methicillin-resistant Staphylococcus aureus among patients and healthcare workers in a Serbian University Hospital. PLoS One 10, e0127347 (2015).

24. Cirkovic, I. D. S., Carevic, B., Mazic, N., Mioljevic, V., Stepanovic, S.: Methicillinresistant Staphylococcus aureus nasal carriage among hospitalized patients and healthcare workers in the Clinical Center of Serbia. Arch Biol Sci 66, 87-92 (2014).

25. Lepsanovic, Z., Jeremic, L. P., Lazic, S., Cirkovic, I.: High prevalence and resistance patterns of community-associated methicillin-resistant Staphylococcus aureus in the Pomoravlje Region, Serbia. Acta Microbiol Immunol Hung 63, 83-92 (2016).

26. Budimir, A., Deurenberg, R. H., Plecko, V., Vink, C., Kalenic, S., Stobberingh, E. E.: Molecular characterization of methicillin-resistant Staphylococcus aureus bloodstream isolates from Croatia. J Antimicrob Chemother 57, 331-334 (2006).

27. Xiao, M., Wang, H., Zhao, Y., Mao, L. L., Brown, M., Yu, Y. S., O'sullivan, M. V., Kong, F., Xu, Y. C.: National surveillance of methicillin-resistant Staphylococcus aureus in China highlights a still-evolving epidemiology with 15 novel emerging multilocus sequence types. J Clin Microbiol 51, 3638-3644 (2013).

28. Masterton, R.: The importance and future of antimicrobial surveillance studies. Clin Infect Dis 47, S21-S31 (2008).

29. Morris, A. K., Masterton, R. G.: Antibiotic resistance surveillance: Action for international studies. J Antimicrob Chemother 49, 7-10 (2002).

30. O'Brien, T. F., Stelling, J.: Integrated multilevel surveillance of the world's infecting microbes and their resistance to antimicrobial agents. Clin Microbiol Rev 24, 281-295 (2011).

31. Alp, E., Leblebicioglu, H., Doganay, M., Voss, A.: Infection control practice in countries with limited resources. Ann Clin Microbiol Antimicrob 10, 36 (2011).

32. Grimshaw, J., Campbell, M., Eccles, M., Steen, N.: Experimental and quasi-experimental designs for evaluating guideline implementation strategies. Fam Pract 17, S11-S16 (2000). 